\title{
Intervenções Percutâneas em Cardiopatias Congênitas
}

\author{
Francisco de Assis Costa, Luiz Junya Kajita, Eulógio Emílio Martinez Filho
}

São Paulo, SP

\begin{abstract}
Os primórdios da cardiologia intervencionista remontam aos idos de 1953, quando Rubio Alvarez e cols. ${ }^{1}$, no México, improvisando um valvótomo, adaptado a uma sonda uretral, dilataram as primeiras valvas pulmonares estenóticas. De lá até os dias atuais, as intervenções percutâneas em cardiologia experimentaram avanços fantásticos, passando pelos primeiros casos de ampliações de comunicações interatriais, publicados em 1966 por Rashkind ${ }^{2}$, pelo pioneiro fechamento de um canal arterial, realizado por Porstmann em $1967^{3}$ e culminando com o advento da angioplastia transluminal coronariana percutânea realizada por Gruentzig ${ }^{4}$, em 1977, o grande impulso para a ocupação definitiva de um espaço cada vez maior dentro da moderna cardiologia. Em 1979, Semb e cols. ${ }^{5}$ realizaram uma valvoplastia pulmonar com cateter angiográfico do tipo Berman, técnica aperfeiçoada por Kan e cols. ${ }^{6}$ que, em 1982, dilataram uma estenose pulmonar valvar em criança de oito anos, no The Johns Hopkins Hospital de Baltimore, utilizando um balão especificamente desenvolvido para esse fim.

Desde então, não param de crescer as indicações do cateterismo intervencionista nas cardiopatias congênitas, seja nas dilatações valvares, nas ampliações de defeitos do septo atrial, nas aortoplastias com ou sem o emprego de endopróteses vasculares (stents), e mais recentemente, na abertura de valvas pulmonares atrésicas, seja nas oclusões dos defeitos dos septos atrial e ventricular e no fechamento do canal arterial pérvio, temas revistos a seguir.
\end{abstract}

\section{Valvoplastia pulmonar}

Generalidades - A estenose pulmonar valvar representa cerca de $10 \%$ de todas as cardiopatias congênitas. $O$ processo é decorrente de espessamento dos folhetos valvares e fusão comissural, conferindo à valva configuração cônica ou em forma de cúpula, com saída estreita em seu

Instituto do Coração do Hospital das Clínicas da FMUSP

Correspondência: Francisco de Assis Costa - InCor - Serviço de Hemondinâmica e Cardiologia Intervencionista - Av. Dr. Enéas C. Aguiar, 44-05403-000 - São Paulo, SP E-mail: fac.smnc@uol.com.b

Recebido para publicação em 9/2/01

Aceito em 7/3/01 ápice ${ }^{7}$. Menos comumente, a estenose pulmonar pode ser causada por uma valva displásica, quando associada a uma forma familiar ou com a síndrome de Noonan ${ }^{8}$. Na valva displásica há acentuado espessamento dos três folhetos sem fusão das comissuras. Os folhetos são, muitas vezes, alongados, estendendo-se até o tronco pulmonar, onde causam obstrução adicional. A estenose pulmonar pode ser, ainda, subvalvar - infundibular ou subinfundibular, sendo que quando do tipo infundibular quase sempre coexiste defeito septal ventricular - e supravalvar, esta decorrente de estreitamento do tronco pulmonar, das artérias pulmonares principais ou periféricas ${ }^{7}$.

Considerações técnicas - Através da veia femoral, introduz-se, via cateter com orifício terminal, fio-guia que deverá ser ancorado em ramo pulmonar periférico distal, tanto quanto possível, a fim de que seja adequado o suporte, para o perfeito manuseio do instrumental. Sobre o fio-guia, leva-se o cateter-balão, a ser posicionado exatamente na altura da valva, sendo aí insuflado até o desaparecimento da indentação nele produzida pela valva estenótica. Durante a insuflação, é comum o surgimento de bradicardia sinusal e extra-sístoles atriais ${ }^{6}$, podendo haver até mesmo o desencadeamento de crises hipoxêmicas por espasmo do infundíbulo hipertrófico. Os melhores resultados são obtidos com o emprego de balões em torno de $20 \%$ a $40 \%$ maiores que o anel valvar.

Indicações - Como, de um modo geral, as crianças são assintomáticas (exceto o recém-nascido com estenose crítica), a indicação de valvoplastia pulmonar tem por base, essencialmente, o gradiente sistólico entre o ventrículo direito e o tronco pulmonar. Quando este gradiente encontra-se em níveis $\geq 50 \mathrm{mmHg}$, valores detectados quer pela ecocardiografia ou pelo cateterismo cardíaco, o procedimento está indicado ${ }^{8-11}$. Mais recentemente, têm-se indicado a valvoplastia pulmonar também em outras cardiopatias congênitas cianogênicas, sobretudo na tetralogia de Fallot, em crianças de baixa idade, com hematócritos elevados e com antecedentes de crises hipoxêmicas, como procedimento de ordem paliativa ou como ponte para a cirurgia corretiva ${ }^{12,13}$.

Resultados-A valvoplastia pulmonar constitui-se, hoje, 
no tratamento de escolha da estenose pulmonar valvar, obtendo-se, na grande maioria dos casos, redução significativa do gradiente sistólico entre o ventrículo direito/tronco pulmonar e, não raro, até mesmo seu desaparecimento. O gradiente residual é, em parte, conseqüência da obstrução representada pelo infundíbulo hipertrófico. O seguimento de longo prazo tem demonstrado a manutenção dos resultados satisfatórios obtidos com a dilatação valvar ${ }^{11,14-16}$. Já, na abordagem da estenose pulmonar da valva displásica, face à pobreza dos resultados alcançados com a valvoplastia, quer pelos elevados gradientes residuais, quer pelo alto índice de reestenose, deve-se optar, preferencialmente, pela correção cirúrgica ${ }^{17,18}$. Na estenose crítica do recém-nascido, quando presente hipoplasia do ventrículo direito e da valva tricúspide, dadas as desfavoráveis condições técnicas e aos resultados nem sempre satisfatórios, é preferível o tratamento cirúrgico ${ }^{16}$.

Complicações - As complicações maiores incluem óbito, perfuração cardíaca e insuficiência tricúspide, todas inferiores a $0,3 \%$, segundo um grande registro multicêntrico. Outras complicações constam de arritmias, edema pulmonar, septicemia, hemorragia no local da punção e trombose da veia femoral ${ }^{11,12,15,19}$.

\section{Valvoplastia aórtica}

Generalidades - A estenose valvar aórtica responde por aproximadamente $3 \%$ a $6 \%$ de todas as cardiopatias congênitas, ou $60 \%$ a $75 \%$ dos casos de estenose aórtica ${ }^{20,21}$. Uma valva aórtica bicúspide é a alteração congênita mais comum a coexistir com a estenose aórtica. Gira em torno de $2 \%$ a incidência de valva aórtica bicúspide na população geral. Nesse tipo de situação verifica-se comissura única, central ou excêntrica e graus variáveis de fusão de suas bordas ${ }^{8,22}$. A estenose aórtica pode ser valvar, supravalvar e subvalvar. A modalidade supravalvar correlaciona-se com estenose da artéria pulmonar e de seus ramos ou pode fazer parte da síndrome de Williams. Apresenta-se sob a forma de membrana, estreitamento em ampulheta, ou sob a forma de hipoplasia da aorta ascendente. Na modalidade subvalvar, o defeito assume forma de membrana, anel fibroso, túnel fibromuscular e de aspecto hipertrófico ${ }^{23}$. Ocasionalmente, pode ocorrer por inserção anômala de cordas da valva mitral no septo interventricular ${ }^{24}$. Coube a Zuhdi Lababidi o mérito de haver realizado, em 1982, a primeira valvoplastia aórtica percutânea em um menino de oito anos, com diagnóstico clínico e ecocardiográfico de estenose aórtica severa. Após a intervenção, houve significativa redução do gradiente transaórtico ( $85 \mathrm{mmHg}$ para $28 \mathrm{mmHg}$ ), na ausência de complicações ${ }^{25}$. Desde então, o procedimento vem sendo realizado com taxas de sucesso que variam de acordo com sua indicação nas mais diversas partes do mundo.

Considerações técnicas - A valvoplastia aórtica é, normalmente, realizada por via retrógrada transarterial. Ultimamente, têm sido descritas técnicas anterógradas por acesso venoso femoral e punção transseptal, com as vantagens de serem evitadas as complicações próprias da aborda- gem arterial, além das dificuldades técnicas da ultrapassagem de uma valva estenótica ${ }^{26}$. A relação balão/anel aórtico deve ser de $1: 1^{8}$. Quando essa relação ultrapassa a marca de 1,2 aumenta de maneira significativa a ocorrência de prolapso e regurgitação valvares ${ }^{21,27}$.

Indicações - Na estenose valvar aórtica da criança, a valvoplastia está indicada quando o gradiente sistólico entre ventrículo esquerdo e a aorta encontra-se acima de $80 \mathrm{mmHg}$, independentemente de sintomas, ou nos casos de gradientes maiores que $50 \mathrm{mmHg}$, se houver sintomas clínicos: evidências de disfunção ventricular esquerda, angina do peito, síncope e pré-síncope ${ }^{8,28}$. Na estenose aórtica crítica do neonato, geralmente manifesta por severa insuficiência cardiocirculatória, a valvoplastia aórtica constitui opção terapêutica a ser considerada, em virtude da alta morbimortalidade associada ao tratamento cirúrgico ${ }^{29-31}$.

Resultados - De um modo geral, observa-se redução significativa do gradiente sistólico transaórtico (gradiente residual entre $20 \mathrm{mmHg}$ e $40 \mathrm{mmHg}$ ), bem como da pressão sistólica intraventricular esquerda ${ }^{32,33}$. Considerando tratar-se de procedimento que pode ser repetido e que a morbimortalidade com a valvoplastia é comparável aos melhores resultados cirúrgicos, sem os inconvenientes da toracotomia e da circulação extracorpórea, a intervenção afigurase, portanto, como opção terapêutica real ao tratamento cirúrgico convencional. Nos casos que cursam com hipoplasia do anel valvar e calcificação valvar severa os resultados são pouco efetivos ${ }^{8}$.

Complicações - De acordo com o registro do NHLBI americano $^{32}$, que consta de 674 valvoplastias aórticas, as complicações relacionadas ao procedimento e até a alta hospitalar estão assim distribuídas: hematomas significantes no sítio de acesso (7\%), necessidade de cirurgia vascu$\operatorname{lar}(5 \%)$, acidentes vasculares cerebrais $(3 \%)$, infarto agudo do miocárdio (3\%), insuficiência aórtica aguda (1\%), necrose tubular aguda $(1 \%)$.

\section{Valvoplastia mitral}

A estenose mitral como seqüela clínica da febre reumática é ainda, e infelizmente, muito prevalente nas nações em desenvolvimento, constituindo-se em verdadeira questão de saúde pública nesses países. A comissurotomia mitral cirúrgica, realizada pela primeira vez em 1925 por Souttar ${ }^{34}$, mantém-se como método eficaz para o tratamento da estenose mitral. No entanto, marcando mais um notável progresso da cardiologia intervencionista, Inoue e cols. ${ }^{35}$ realizaram no Japão, em 1984, a primeira comissurotomia mitral percutânea, utilizando balão único, técnica que nos dias de hoje leva o seu nome. Em 1986, AlZaibag ${ }^{36}$, na Arábia Saudita, foram os primeiros a relatar uma valvoplastia mitral empregando a técnica do duplo balão. Essas duas técnicas rapidamente se difundiram e são hoje em dia amplamente praticadas em todas as latitudes do planeta. Suas indicações e resultados na estenose mitral do adulto são sobejamente conhecidos e fogem dos objetivos deste capítulo.

A estenose mitral congênita ocorre em $0,2 \%$ a $0,5 \%$ dos 
pacientes com defeitos congênitos e pode ser responsável por profundas alterações hemodinâmicas na criança ${ }^{37,38}$. São reconhecidos quatro tipos básicos de defeitos anatômicos: 1) estenose mitral típica, com espessamento dos folhetos, encurtamento das cordas tendíneas e espaços intercordais diminuídos;2) membrana supravalvar mitral; 3) valva mitral em pára-quedas e 4) síndrome do coração esquerdo hipoplásico ${ }^{39}$. Conforme já mencionado, a valvoplastia mitral tem sido empregada com sucesso em adultos com estenose mitral de origem reumática. Contudo, as informações a respeito do procedimento na estenose mitral congênita ainda são escassas ${ }^{40,41}$. Grifka e $\operatorname{cols}^{38}$. relataram que a técnica do duplo balão foi eficaz na redução do gradiente transmitral e no aumento da área valvar em oito pacientes, tendo sido registrado apenas um caso de insuficiência mitral $(++/ 4+)$. Há outros relatos favoráveis também com a técnica de Inoue ${ }^{42-44}$. A intervenção cirúrgica em crianças, seja a comissurotomia ou a troca valvar, cursa com elevada mortalidade operatória: $17 \%$ a $19 \%$ e $25 \%$ a $40 \%$, respectivamente ${ }^{43}$. De acordo com os dados disponíveis, o procedimento percutâneo tem sido eficaz, é mais seguro e dispensa a toracotomia. Por outro lado, a valvoplastia mitral por cateter-balão para tratamento da estenose mitral reumática em crianças tem apresentado excelentes resultados. Fawzy e cols. ${ }^{42}$ descreveram dilatação com balão de Inoue em 30 pacientes com idade entre 10 e 18 anos. Cotejando-se os resultados do procedimento em crianças menores de 12 anos com aqueles obtidos em adultos, observou-se maior aumento da área valvar no primeiro grupo, sem aumento adicional dos riscos.

\section{Valvoplastia tricúspide}

A estenose da valva tricúspide, de infreqüente diagnóstico clínico, tem como causas a síndrome carcinóide, a febre reumática, anormalidades congênitas e endocardite infecciosa. A estenose congênita resulta de defeitos de um ou mais dos elementos do aparelho valvar (folhetos, cordas tendíneas e músculos papilares), a exemplo do que ocorre com a estenose mitral congênita ${ }^{8}$. A estenose tricúspide encontra-se presente no exame anátomo-patológico de $5 \%$ a $10 \%$ dos pacientes com cardiopatia reumática, quase sempre associada a envolvimento das valvas mitral e aórtica ${ }^{45}$. Entretanto, a estenose tricúspide é seqüela clínica rara da febre reumática, estando presente em apenas 3\% a 5\% dos portadores de estenose mitral reumática ${ }^{46}$.

A comissurotomia tricúspide foi pioneiramente realizada por Alvarez e Lason em 1955, no México ${ }^{47}$. Já a valvoplastia tricúspide por cateter balão vem sendo praticada desde 1987, a partir da experiência inicial de Al Zaibag e cols. ${ }^{48}$. As técnicas utilizadas têm sido, como na valvoplastia mitral, a de Inoue e a do duplo balão ${ }^{49-53}$. De lá para cá, no entanto, acumulam-se relatos isolados de dilatação da valva tricúspide, que, a julgar pelas altas taxas de sucesso primário, melhora dos parâmetros clínicos e hemodinâmicos e baixas complicações, representadas sobretudo pelos distúrbios da condução atrioventricular, poderá o método constituir-se em alternativa ao tratamento cirúrgico convencional ${ }^{49-53}$.

\section{Aortoplastia na coarctação da aorta}

Generalidades - A coarctação da aorta representa aproximadamente $6 \%$ das cardiopatias congênitas ${ }^{24}$. Há nítida associação com valva aórtica bicúspide, anomalias congênitas da valva mitral, fibroelastose endocárdica, canal arterial pérvio, defeito septal ventricular, além da relação com a síndrome de Turner e com o complexo de Shone. Caracteriza-se por estreitamento situado na aorta descendente, após a croça aórtica, classificando-se em pré-ductal, justaductal e pós-ductal, conforme sua posição em relação ao canal arterial ${ }^{54}$. A zona de coarctação localiza-se, amiúde, adiante da artéria subclávia esquerda, cuja porção proximal acha-se dilatada ${ }^{55}$. As primeiras tentativas de dilatação da aorta tiveram início em 1982, quando Lock e cols. ${ }^{56}$ demonstraram os efeitos do balão sobre segmentos excisados de aorta. Desde então, seguiram-se inúmeras outras publicações ${ }^{57-62} \mathrm{e}$, nos dias atuais, a aortoplastia com ou sem a utilização de stents vem-se estabelecendo como potencial opção terapêutica no tratamento dessa cardiopatia congênita.

Considerações técnicas - A abordagem é arterial por via retrógrada. O centro do balão deverá estar posicionado exatamente no local a ser dilatado. Não deve exceder de $0,9 \mathrm{a}$ 1,0 a relação entre o diâmetro do balão e diâmetro de referência do segmento aórtico comprometido. Quanto aos locais utilizados, como referência para a medida do balão, consideram-se a área adjacente à coarctação (preferida no Instituto do Coração), o segmento aórtico na altura do diafragma e aquele situado junto à artéria subclávia esquerda ${ }^{63}$. O mecanismo de dilatação é o resultado da ruptura e dissecção da camada íntima arterial, o que explica a eventual formação de aneurismas ${ }^{57,61,64}$.

Indicações - A aortoplastia por balão pode ser indicada na coarctação nativa da aorta (estenoses anulares, concêntricas) e nas recoarctações. São critérios clínicos para a indicação do procedimento, a presença de hipertensão arterial sistêmica e de insuficiência cardíaca congestiva, principalmente em neonatos. Outro parâmetro de utilidade é a existência de estenose igual ou maior que $50 \%$ da luz do vaso, diagnosticada pela ecocardiografia ou pela aortografia ${ }^{28,65}$. O gradiente pressórico entre as porções pré e pós-coarctação carece de confiabilidade, pois sofre interferência da rede colateral que se forma, a depender da magnitude da obstrução.

Resultados - Atualmente, a aortoplastia por balão tem sido o tratamento de escolha nas recoarctações, onde a formação fibrosa no local e nas adjacências da excisão cirúrgica ou na área previamente dilatada confere certa proteção contra o trauma provocado pela (re)insuflação do balão ${ }^{63}$. No que concerne à coarctação nativa, a intervenção deve ser avaliada, levando-se em conta a faixa etária do paciente. Em neonatos e lactentes, em face das altas taxas de reestenose a curto prazo (até 90\%), a indicação de aortoplastia 
deve ser restrita aos casos que cursam com grave insuficiência cardíaca congestiva, tendo, portanto, caráter paliativo. Em crianças acima de um ano de idade, adolescentes e adultos (exceto idosos), os resultados têm-se mostrado excelentes, fortalecendo uma tendência de indicar-se o procedimento como o de escolha no tratamento da coarctação nesse grupo etário, com taxas de sucesso imediato superiores a $90 \%$, incidência de aneurismas entre $1,9 \%$ e $4,5 \%$ e reestenose entre $10 \%$ e $20 \%{ }^{61}$. São considerados critérios indicativos de sucesso: gradiente residual $<20 \mathrm{mmHg}$, área dilatada angiograficamente livre de obstrução e normalização da pressão arterial em membros superiores. São fatores preditores de reestenose: hipoplasia do istmo aórtico, gradiente residual $>12 \mathrm{mmHg}$ e valor da relação diâmetro do istmo/diâmetro da aorta descendente $<0,65^{61,65}$.

Complicações - Complicações vasculares no sítio de acesso, sobretudo em crianças de tenra idade, acidente vascular cerebral, isquemia abdominal, crise hipertensiva, aneurismas no segmento dilatado e rotura da aorta têm sido relatados em diversas séries ${ }^{57-62,64,66,67}$.

\section{Atriosseptostomia}

Generalidades - A atriosseptostomia por balão teve início em 1962 com Rashkind ${ }^{2}$, objetivando a ampliação de comunicações interatriais existentes em algumas malformações cardíacas para assim proporcionar maior mistura entre as circulações pulmonar e sistêmica, com o conseqüente aumento da saturação sangüínea de oxigênio. Paralelamente, o incremento do fluxo interatrial, elevando o débito sistêmico e diminuindo a carga pressórica sobre o leito venoso pulmonar, constitui o outro pilar fisiopatológico que sustenta a indicação desse procedimento paliativo nas cardiopatias descritas a seguir. Com similar intento, desde $1975^{68-69}$ passou-se a utilizar cateter com lâmina para atriosseptostomia de crianças fora do período neonatal (idade $>1$ mês de vida) e em outras situações nas quais é maior a rigidez do septo atrial, a partir de trabalho pioneiro de Garcia e cols. $^{70}$, em 1973.

Considerações técnicas - Através de via venosa femoral, o cateter-balão é avançado até o átrio direito, ultrapassando a comunicação interatrial e posicionado na altura de uma veia pulmonar, preferencialmente na veia pulmonar superior esquerda, onde é insuflado e gentilmente recuado até o exato nível da comunicação, onde deve ser tracionado de forma curta e vigorosa, para que o balão atinja apenas o septo interatrial e não alcance a veia cava inferior. Deve-se ter especial atenção no momento do recuo do balão da veia pulmonar até o septo. Nesta fase, um pequeno deslocamento do balão poderá posicioná-lo na altura da valva mitral, com conseqüências desastrosas, quando tracionado inadvertidamente. Com relação ao posicionamento do cateter com lâmina, aplicam-se as considerações técnicas aludidas para o cateter-balão, o qual deverá, na medida do possível, complementar a ampliação conseguida pelo cateter com lâmina.

Indicações - Fundamentalmente norteadas por critéri- os clínicos, isto é, cianose, retratando graus variáveis de hipoxemia e/ou insuficiência cardíaca congestiva. O protótipo da indicação para a realização de atriosseptostomia por balão era a transposição das grandes artérias com comunicação interatrial restritiva. Atualmente, as indicações foram estendidas e incluem outras cardiopatias nas quais haja a necessidade de uma grande comunicação interatrial, permitindo uma melhor saturação sangüínea de oxigênio, tais como atresia tricúspide, atresia pulmonar com septo ventricular íntegro, dupla via de saída do ventrículo direito com comunicação interatrial e interventricular restritivas, hipoplasia do coração esquerdo e do coração direito, atresia mitral ou estenose mitral congênita severa e drenagem anômala total das veias pulmonares. Já a atriosseptostomia por cateter com lâmina encontra indicação em determinadas circunstâncias, onde é limitada a eficácia do balão, tendo em vista a maior rigidez do septo atrial, como crianças acima de um mês de vida e em algumas cardiopatias em que há, habitualmente, espessamento anormal do septo atrial ${ }^{68-70}$, como na atresia mitral, dupla via de saída do ventrículo direito com estenose mitral e ventrículo único com estenose mitral. Convém ressaltar, que a atriosseptostomia por balão deve, sempre que possível, complementar a ampliação obtida pelo cateter com lâmina.

Resultados - Observadas as indicações mencionadas referidas, trata-se de procedimento paliativo que se segue de dramática e substantiva melhora da saturação arterial de oxigênio, da acidose metabólica ou do quadro congestivo, conforme tenha sido a indicação do procedimento, propiciando, dessa maneira, sobrevida menos penosa para o infante, até que seja instituído o tratamento definitivo. Em se tratando de critério hemodinâmico, admite-se que o gradiente pressórico médio entre os dois átrios deva ser $\leq 3 \mathrm{mmHg}$ para que a atriosseptostomia seja considerada efetiva.

Complicações - Arritmias cardíacas, laceração da veia cava inferior e da valva mitral com conseqüente regurgitação mitral, tamponamento cardíaco e morte, cuja incidência é muito baixa, quando o procedimento é realizado por profissional habilitado a executá-lo.

\section{Abertura da valva pulmonar atrésica}

A atresia pulmonar com septo ventricular íntegro corresponde a aproximadamente $2,5 \%$ de todas as cardiopatias congênitas, sendo a segunda causa de severa hipoxemia no $1^{\circ}$ mês de vida, superada apenas pela transposicão das grandes artérias ${ }^{71}$. Como não se trata de anomalia isolada, pois o ventrículo direito e a valva tricúspide são, via de regra, também acometidos, o tratamento cirúrgico continua a ser um desafio, em face da hipoplasia do ventrículo direito, presente em graus variáveis ${ }^{72}$. Há evidente correlação entre o anel valvar tricúspide e o tamanho do ventrículo direito, parâmetro utilizado para se proceder à escolha do tratamento cirúrgico mais apropriado. Sendo o ventrículo direito bem desenvolvido, opta-se pela abertura cirúrgica (técnica de Brock), seguida de um shunt sistêmico-pulmonar do tipo Blalock-Taussig. Por outro lado, se o ventrículo direito é 
moderadamente desenvolvido, a opção preferencial tem sido a cirurgia de Glenn bidirecional. Já nos casos que se acompanham de hipoplasia extrema do ventrículo direito, a escolha deve recair sobre a cirurgia de Fontan.

A partir de 1990, porém, a valva pulmonar atrésica acompanhada de ventrículo direito bem desenvolvido, passou a contar com uma nova alternativa para o seu tratamento, por intermédio do cateterismo cardíaco, desde a experiência inicial de Qureshi e cols. ${ }^{73}$, que relataram casos de perfuração valvar bem sucedida em quatro de cinco crianças selecionadas para o procedimento, realizado através de um cateter, acoplado a um gerador de energia laser. A intervenção foi complementada por valvoplastia pulmonar convencional com cateter-balão. Todavia, apesar dos bons resultados, o alto custo operacional, complexidade técnica, e possibilidade de maior dano tecidual, acarretando maiores complicações, foram algumas das causas que vieram a inviabilizar o uso da técnica. Desse modo, seguindo o firme propósito de restabelecer a continuidade ventrículo direito/ artéria pulmonar, via cateterismo intervencionista, Hausdorf e cols. ${ }^{72}$ publicaram, em 1993, o primeiro relato de abertura de uma valva pulmonar atrésica (do tipo muscular), utilizando um cateter que possui, em sua extremidade, um eletrodo de liga metálica que se aquece tão logo seja estimulado pela energia liberada do gerador de radiofreqüência, obtendo os autores sucesso na perfuração da valva, procedimento concluído com valvoplastia por balão e implante de stent. Ainda naquele ano, os mesmos pesquisadores publicaram uma série contendo 28 casos de perfuração de valva pulmonar atrésica, usando o princípio da radiofreqüência, tendo 15 desses pacientes atresia do tipo muscular e 13 do tipo membranoso ${ }^{74}$. A dilatação complementar por balão foi efetuada em todos os 28 pacientes e em oito deles houve necessidade de implante de stent. Sobre implante de stents, aliás, usados principalmente para manter a perviabilidade valvar nas atresias do tipo muscular, cabe a ressalva de que a presença da prótese na região infundibular do ventrículo direito é potencial geradora de graves arritmias ventriculares. Há outros relatos favoráveis com o uso da técnica ${ }^{75,76}$. No Brasil, Fontes e cols. ${ }^{77}$ publicaram, em 1995, o primeiro relato de perfuração valvar, realizada em um recém-nascido, portador de atresia pulmonar do tipo membranoso, logrando êxito com a intervenção.

Em portadores de atresia pulmonar com septo ventricular íntegro, a mortalidade hospitalar com cirurgias paliativas é de aproximadamente $10 \%$. Se forem consideradas outras intervenções cirúrgicas para correção definitiva, essa taxa pode atingir níveis de $25 \%$ a $50 \%$ de mortalidade hospitalar ${ }^{73}$. Feitas tais considerações, somadas ao fato de que a perfuração valvar por radiofreqüência é de baixo custo operacional, simplicidade técnica e diminui sobremodo a possibilidade de danos teciduais, quando comparada ao laser, trata-se de procedimento que poderá ser incorporado ao cotidiano dos laboratórios de hemodinâmica, para tratamento de casos selecionados de atresia pulmonar, sobretudo do tipo membranoso, que tenham ventrículo direito moderadamente ou bem desenvolvidos. A atresia infundi- bular e a agenesia do tronco pulmonar são contra-indicações para o emprego do método ${ }^{77}$.

\section{Oclusão do canal arterial pérvio}

Generalidades - O canal arterial, fundamental na circulação fetal, normalmente fecha após o nascimento, verificando-se sua oclusão funcional nas primeiras $10-15 \mathrm{~h}$ de vida, com a oclusão anatômica completando-se entre a $2^{\mathrm{a}}$ e a $3^{\mathrm{a}}$ semanas de vida ${ }^{24}$. Ocorre como defeito isolado em $0,01 \%$ a $0,08 \%$ de todos os nascidos vivos, representando cerca de $6 \%$ a $11 \%$ do total das cardiopatias congênitas ${ }^{78,79}$. Foi a primeira cardiopatia congênita a ser corrigida cirurgicamente, em 1938, por Gross e Hubbard ${ }^{80}$, além de ter sido, também, a primeira a ser ocluída por método percutâneo, em 1967, por Porstmann ${ }^{3}$. Segundo a classificação anatômica de Krichenko e cols. ${ }^{81}$, que tem como referência o estreitamento do ductus, sua relação com a aorta e com a artéria pulmonar, bem como com a sombra radiológica da traquéia, o canal arterial pode pertencer ao grupo " $\mathrm{A}$ " (o mais comum), cujo formato é cônico e a zona de estreitamento relaciona-se com a artéria pulmonar (subdividido em A1, A2 e A3 conforme a relação da estenose com as porções anterior, central ou posterior da sombra radiológica da traquéia); grupo "B", no qual o canal é curto (em janela) e o estreitamento relacionase com a inserção aórtica (subdividido em B1, B2 e B3, utilizando o mesmo critério que relaciona a estenose à sombra traqueal); grupo "C", no qual o canal é tubular e não existe estreitamento. No grupo "D", o canal apresenta múltiplas estenoses e, finalmente, no grupo "E" o canal assume formas bizarras.

Considerações técnicas-Porstmann, em 1967, realizou a primeira oclusão percutânea de canal arterial pérvio utilizando um plug de ivalon. Apesar dos bons resultados, o método vem perdendo espaço para outros (umbrellas e coils), em vista das limitações de idade e peso, além de necessitar de um cateter liberador de grosso calibre, que requer artéria femoral de diâmetro compatível (em média $3,0 \mathrm{~mm}$ ), o que nem sempre é possível ${ }^{82}$.

Sobre a umbrella de Rashkind-Depois de modificações progressivas, atualmente a umbrella dupla de Rashkind é constituída de dois discos de poliuretano, sustentados por uma armação de aço inoxidável com três ou quatro hastes, consoante seja a umbrella tamanho $12 \mathrm{~mm}$ ou $17 \mathrm{~mm}$. A umbrella pode ser implantada por via arterial ou venosa, sendo a última preferível pela simplicidade e segurança técnicas. Com um cateter pela via venosa cruza-se o canal arterial, deixando-se um outro, tipo pigtail no plano da valva aórtica para realização de aortografia e visibilização angiográfica do canal, além de monitorização pressórica. A sombra radiológica da traquéia também é utilizada como referência para a localização do canal arterial. O sistema de liberação é controlado por cateter especialmente desenvolvido para o implante. Bainhas de Mullins longas 8F (french) são necessárias para o implante de umbrellas tamanho $12 \mathrm{~mm} \mathrm{e}$ bainhas $11 \mathrm{~F}$ para umbrellas tamanho $17 \mathrm{~mm}$. A prótese tamanho $12 \mathrm{~mm}$ deve ser empregada para fechamento de canais 
com diâmetro de até $4 \mathrm{~mm}$, reservando-se o dispositivo de $17 \mathrm{~mm}$ para canais com diâmetro entre $4 \mathrm{e} 8 \mathrm{~mm}^{83-85}$.

Sobre molas espirais (coils) - A oclusão de canal arterial pérvio com os chamados coils de Gianturco foi realizada pela primeira vez por Cambier e cols. ${ }^{78}$, com publicação em 1992. O método apresenta algumas vantagens em relação aos demais: simplicidade técnica, permitindo mais fácil aprendizado, uso de introdutores de pequeno calibre e menor custo operacional. Através da via arterial, introduz-se cateter tipo Judkins (de coronária direita) $5 \mathrm{~F}$ ou $6 \mathrm{~F}$ ou outro modificado que deverá ser posicionado no tronco pulmonar. Em seguida, a mola em espiral é passada por dentro do cateter e empurrado por fio-guia metálico 0,035 " ou 0,038 " até o tronco pulmonar. Após formada a primeira alça o cateter é suavemente recuado até que essa alça ancore no coto pulmonar, prosseguindo-se com o recuo do cateter até que o coil esteja completamente exteriorizado. Para a adequada oclusão do pertuito, pelo menos três alças devem permanecer formadas no interior do canal, preferencialmente quatro ou cinco alças. Conseguida a completa liberação do coil com oclusão do ductus, um aortograma na projeção perfil esquerdo deverá ser repetido $15 \mathrm{~min}$ após ${ }^{85-87}$.

Indicações - Uma vez diagnosticado, o canal arterial pérvio deve ser ocluído, quer cirurgicamente, quer por intervenção percutânea. Abaixo do $1^{\circ}$ ano de vida devem ser tratadas as crianças que apresentam evolução clínica desfavorável: insuficiência cardíaca congestiva, pneumonias de repetição, sinais de hipertensão arterial pulmonar, déficit pôndero-estatural acentuado. Os candidatos a procedimento com umbrella devem, idealmente, ter peso superior a $8 \mathrm{~kg}$ e canais com diâmetro de até $8 \mathrm{~mm}^{83-85}$. Para fechamento com coils, as melhores indicações recaem sobre os canais dos grupos "A", "D" e "E" de Krichenko com diâmetro de até 2,5-3,5mm. O diâmetro interno do canal deve ser $\leq 50 \%$ do diâmetro do coil ${ }^{85-87}$.

Resultados - Os percentuais de sucesso em relação ao fechamento do canal arterial, tanto para os procedimentos com umbrella, quanto para os realizados com coils, superam a casa dos $90 \%$. Os canais de maior diâmetro e aqueles dos grupos "B" e "C" da classificação anatômica de Krichenko têm-se revelado como preditores de insucesso para o implante de ambos os dispositivos ${ }^{85}$. No tocante à intervenção com umbrella, Hosking e cols. ${ }^{88}$, em estudo que acompanhou a evolução precoce e tardia de 177 pacientes submetidos ao implante da prótese, incluindo também aqueles que receberam uma segunda umbrella, relataram a prevalência de shunt residual da ordem de $53 \%$ um dia após o implante, $38 \%$ após um ano, $18 \%$ após dois anos e $8 \%$ ao final de 40 meses. Já no registro europeu sobre implante de umbrellas, incluindo 686 pacientes (taxa de sucesso de $94,8 \%$ ), a prevalência global de shunts residuais, considerando pacientes que receberam um ou dois dispositivos, foi de 5,2\% após 30 meses de seguimento ${ }^{89}$. Por outro lado, ratificando os resultados de vários trabalhos ${ }^{86,87,90,91}$, um grande registro multicêntrico sobre fechamento de canal arterial com coils, constando de 535 pacientes, relatou suces- so de implante em $95 \%$ dos casos, oclusão completa (ao doppler) nas primeiras $24 \mathrm{~h}$ em $75 \%$, com oclusão parcial nos demais $20 \%{ }^{92}$.

Complicações - Com relação à umbrella, as complicações incluem embolização da prótese (2,0\% a 2,4\%), hemólise $(0,5 \%$ a l, $0 \%)$, além da possibilidade de estenose da artéria pulmonar esquerda e endarterite infecciosa ${ }^{89,93,94}$. Para os coils, as complicações mais comumente relatadas são embolização (3\% a 20\%), complicações arteriais no sítio de punção (diminuição de pulso e hematoma em cerca de $2 \%$ dos casos), hemólise, obstrução da artéria pulmonar esquerda e pseudo-coarctação da aorta $85,87,92,94,95$.

Nota: Masura e cols. ${ }^{96}$, em estudo com 24 pacientes submetidos a oclusão de canal arterial pérvio, utilizando uma nova prótese de Amplatzer desenvolvida para essa finalidade, relataram sucesso de implante em $100 \%$ dos casos, ausência de shunt residual ao doppler de $24 \mathrm{~h}$, assim como de complicações. Ademais, o dispositivo pode ser implantado através de cateteres $5 \mathrm{~F}$ ou $6 \mathrm{~F}$ e mostrou-se eficaz para fechamento de canais com diâmetro de até $6,0 \mathrm{~mm}$. De acordo, portanto, com esse estudo preliminar, a nova prótese apresenta-se como excelente proposta para o definitivo tratamento do canal arterial pérvio.

\section{Oclusão percutânea da comunicação interatrial}

Generalidades - A comunicação interatrial responde por aproximadamente $7 \%$ a $11 \%$ de todas as cardiopatias congênitas, podendo ser da variedade ostium secundum (a mais comum), ostium primum (quase sempre associada a fendas na cúspide anterior da valva mitral), e seio venoso, relacionada à drenagem anômala parcial das veias pulmonares ${ }^{24,97}$. A depender do tamanho da comunicação, o índice de fechamento espontâneo da comunicação interatrial exibe enorme variação, com publicações na literatura relatando percentuais que oscilam entre $0 \%$ e $66 \%{ }^{98-101}$. Com efeito, apesar de o primeiro relato de oclusão percutânea de uma comunicação interatrial datar de 1974, a partir da experiência inicial de King e Mills ${ }^{102}$, ainda nos dias atuais a cirurgia convencional tem sido o principal instrumento de correção do defeito. Entretanto, o expressivo avanço dos recursos tecnológicos, aliado aos resultados alcançados pela cardiologia intervencionista no tratamento de várias outras cardiopatias congênitas, vêm revigorando as atenções pela correção não cirúrgica dessa afecção congênita.

Indicações - Tendo presente limitações técnicas ainda não vencidas, tão-somente o tipo ostium secundum de comunicação interatrial, ou seja, aquelas situadas na fossa oval podem ser abordadas percutaneamente. A propósito, deve-se proceder à análise percuciente, com vistas à exclusão de outros tipos de comunicação interatrial, assim como de cardiopatias associadas que requeiram cirurgia. Estritamente com base em aspectos clínico-hemodinâmicos, três situações encontram indicação para a intervenção percutânea na comunicação interatrial: 1) aquelas com repercussões clínicas e/ou hemodinâmicas, isto é, presença de sinais ou sintomas clínicos, sobrecarga de volume em câmaras 
direitas e relação entre fluxo pulmonar/fluxo sistêmico $\geq 1,5 ; 2$ ) presumível ou constatada embolia paradoxal; 3) comunicação interatrial pós-cirúrgica, como as existentes no Fontan fenestrado ${ }^{103}$.

Considerações técnicas - Desde o relato pioneiro de King e Mills, até o ressurgimento do interesse pelo fechamento percutâneo da comunicação interatrial no final da década de 80 , várias foram as próteses usadas para a correção do defeito através do cateterismo cardíaco. Assim, surgiram as próteses Clamshell $I \mathrm{e} I I$, derivadas da prótese de Rashkind, Rashkind PDA umbrella, buttoned device, entre outras, cuja complexidade técnica de implante e resultados variam conforme o método utilizado. Todavia, a despeito de relatos favoráveis ${ }^{104-107}$, nenhum dos devices citados conseguiu se firmar como alternativa de fato para o tratamento não-cirúrgico da comunicação interatrial ${ }^{108-111}$. A prótese de Amplatzer surgiu recentemente e parece ser um recurso promissor para a correção percutânea da comunicação interatrial. O dispositivo é constituído de dois discos auto-expansíveis unidos por uma espécie de eixo central que deve corresponder ao diâmetro estirado da comunicação interatrial. O conjunto é composto de uma malha de nitinol, revestida em seu interior por fragmentos de poliéster, visando assim a ampliar sua trombogenicidade. As próteses são disponíveis nos tamanhos de 4 a $26 \mathrm{~mm}$ (diâmetro do eixo central). Os discos são maiores que o eixo em $16 \mathrm{e} 10 \mathrm{~mm}$, respectivamente, para os discos distal e proximal. Devem ser consideradas para oclusão com o dispositivo de Amplatzer comunicações com até $20 \mathrm{~mm}$ de diâmetro ${ }^{109,111}$.

Resultados-Na experiência com a prótese de Amplatzer, de acordo com várias séries publicadas, a oclusão do defeito sem shunt residual imediato varia de $81 \%$ a $100 \%{ }^{107,108,111}$. Mesmo nos casos que não se acompanham de fechamento completo da comunicação interatrial, os shunts residuais foram reconhecidos como triviais, desaparecendo no seguimento tardio, após o $3^{\circ}$ mês de implante do dispositivo. A cirurgia convencional é recurso eficaz e de baixa mortalidade (cerca de 1\%). A julgar, todavia, pelos resultados preliminares obtidos com o dispositivo de Amplatzer, consubstanciados pelas altas taxas de sucesso primário e complicações praticamente inexistentes, o método, eliminando os inconvenientes da toracotomia e da circulação extracorpórea, diminuindo o tempo de internação e custos hospitalares, reacende as expectativas do encontro de uma maneira simples, segura e eficaz para o tratamento não-cirúrgico dessa anormalidade cardíaca tão freqüente entre as cardiopatias congênitas.

Complicações - Segundo os diversos estudos publicados, as complicações geralmente relatadas são embolização da prótese, ataque isquêmico transitório, taquicardia paroxística supraventriculare distúrbios da condução atrioventricular ${ }^{109,111,112 .}$

\section{Oclusão da comunicação interventricular}

De acordo com sua localização no septo ventricular, a comunicação interventricular pode ser do tipo perimembra- noso (a mais freqüente), muscular, de via de entrada e de via de saída ${ }^{113}$. Excluída a valva aórtica bicúspide, trata-se do defeito congênito de maior ocorrência na população geral, sendo responsável por cerca de $20 \%$ do total das cardiopatias congênitas, refletindo uma incidência de $1,5 \%$ a 2,5\% por 1.000 nativivos ${ }^{114}$. A exemplo do que acontece com o canal arterial pérvio, a correção cirúrgica no $1^{\circ}$ ano de vida deve restringir-se aos casos de grandes comunicações, que cursam com insuficiência cardíaca congestiva, infecções pulmonares repetitivas, hipertensão arterial pulmonar e importante déficit pôndero-estatural. Excetuadas essas situações e levando-se em conta as altas taxas de fechamento espontâneo, especialmente nos pequenos defeitos, que podem alcançar o percentual de quase $70 \%$ no $1^{\circ}$ ano de vida, observando-se fechamento espontâneo de moderados ou mesmo de grandes defeitos até que a criança complete 8-10 anos de idade ${ }^{115}$, é prudente e de bom alvitre a conduta expectante com acompanhamento clínico durante todo esse período. A partir dessa fase, são controversas as opiniões sobre a melhor conduta a ser adotada. Alguns defendem o seguimento clínico, tendo em vista o risco cirúrgico (em ge$\mathrm{ral}<1 \%$ ), outros propugnam o tratamento cirúrgico, alegando o risco de endocardite infecciosa (1,5/1.000/paciente/ ano) ${ }^{116}$, além das conseqüências psico-sociais que só o sabem aqueles que carregam consigo um sopro audível no peito. Na tentativa de oferecer uma outra opção de tratamento desse defeito de tão comum ocorrência, Lock e cols. ${ }^{117}$ publicaram, em 1988, os primeiros relatos de oclusão percutânea de comunicação interventricular, utilizando $u m$ brella, série que constava de seis pacientes, sendo três portadores de comunicação interventricular pós-infarto agudo do miocárdio. Sucederam-se outras publicações a respeito ${ }^{118,119}$. Recentemente, Sideris e cols. ${ }^{120}$ relataram experiência positiva na oclusão percutânea de comunicação interventricular com o buttoned device. Foram selecionados 18 pacientes (idade variando de 4 a 35 anos), portadores de comunicação interventricular do tipo membranoso, submetidos ao implante do dispositivo. O procedimento foi guiado por ecocardiografia e por fluoroscopia. Dos 18 pacientes tratados, houve 13 oclusões completas e os demais apresentaram shunts residuais considerados triviais e apenas um paciente desenvolveu bloqueio atrioventricular de $3^{\circ}$ grau transitório. Aguardam-se informações a respeito do seguimento tardio. Por sua vez, Thanopoulos e cols. ${ }^{121}$ relataram série composta de 6 pacientes (idades entre 3 e 10 anos), submetidos a oclusão de comunicação interventricular através de prótese de Amplatzer modificada. A intervenção também foi guiada por ecocardiografia (transesofágica) e por fluoroscopia. Verificou-se êxito em todos os casos tratados e apenas dois pacientes apresentaram bloqueio de ramo esquerdo transitório. Como na série anteriormente referida, resta saber qual o comportamento dessas próteses no seguimento tardio. Contudo, tomando por base os resultados atingidos nas séries até então publicadas, infere-se que o procedimento é eficaz, seguro e que, doravante, poderá surgir como alternativa para o tratamento não-cirúrgico de comunicação interventricular, em casos selecionados. 


\section{Endopróteses vasculares (stents)}

Primariamente concebidos para uso intracoronariano ${ }^{122-124}$, os stents cruzaram fronteiras e têm sido também utilizados em cardiopatias congênitas. Em 1994, foram publicados dois trabalhos experimentais ${ }^{125,126}$ sobre o emprego dessas próteses em animais com coarctação aórtica cirurgicamente produzida, cujos positivos resultados preliminares abriram novos horizontes para o seu implante em aortas coarctadas de seres humanos, fato que pôde ser constatado pelas muitas publicações que se seguiram ${ }^{127-132}$. Como se sabe, em algumas cardiopatias congênitas a necessidade de manutenção da perviabilidade de certas estruturas cardiovasculares, às vezes fundamentais à sobrevivência, sejam elas naturais ou artificiais, descortina a perspectiva do uso cada vez maior desses dispositivos em determinados defeitos congênitos. Assim, avolumam-se na literatura informações acerca do implante de stents nas mais variadas situações patológicas: artérias pulmonares estenóticas, veias pulmonares (na drenagem anômala das veias pulmonares com obstrução), na manutenção do canal arterial pérvio em crianças que precisam desse pertuito aberto para permanecerem vivas, no restabelecimento da continuidade ventrículo direito/tronco pulmonar, nas anastomoses sistêmico-pulmonares estenóticas, entre outras ${ }^{133-139}$. Neste contexto, de acordo com os inúmeros estudos publicados, parece razoável considerar que já existe lugar assegurado para os stents como tratamento paliativo de algumas dessas afecções congênitas. No entanto, a necessidade de reexpansão das próteses na população pediátrica, além do seu comportamento a médio e longo prazos, no que se refere a obstrução ou reestenose, são alguns dos fatores que definirão o exato papel desses dispositivos, como opção real a ser incorporada aos tantos recursos já existentes para propiciar melhor sobrevida à criança portadora de anomalias cardíacas congênitas.

\section{Referências}

1. Rubio-Alvarez V, Limon-Lason R, Soni J.Valvulotomias intracardiacas por medio de un cateter. Arch Inst Cardiol Méx 1953; 23: 83-192.

2. Rashkind WJ, Miller WW. Creation of an atrial septal defect without thoracotomy: a palliative approach to complete transposition of the great arteries. JAMA 1966; 196: 991-2.

3. Porstmann W, Wierny L, Warnke H. Der verschluss des ductus arteriosus persistentens ohne thorakotomie. Thorax Chirurgie 1967; 15: 199-203.

4. Gruentzig A.Transluminal dilatation of coronary artery stenosis. Lancet 1978; 1: 263.

5. Semb BKH, Tjonneland S, Stake G, Aabyholm G. Ballon valvotomy of congenital pulmonary valve stenosis with tricuspid insufficiency. Cardiovasc Radiol 1979; 2: 239-41.

6. Kan JS, White Jr RI, Mitchell SE, Gardner TJ. Percutaneous ballon valvuloplasty: a new method for treating congenital pulmonary valve stenosis. N Engl J Med 1982; 307: 540-2.

7. PerloffJK. Estenose pulmonar congênita. In: PerloffJK. O Reconhecimento Clínico das Cardiopatias Congênitas. $3^{\text {a }}$ Ed. São Paulo: Livraria Editora Santos, 1987: 193.

8. Kajita LJ, Takimura CK. Valvotomias na infância e em neonatos. Rev Soc Cardiol Estado de São Paulo 1995; 5: 481-5.

9. Schneeweiss A, Blieden LC, Shem-Tov A, Goor D, Milo S, Neufeld HN. Diagnostic angiocardiographic criteria in displastic stenotic pulmonic valve. Am Heart J 1983; 106: 761-2.

10. Papadopoulos G, Folger Jr GM. Progressive pulmonary arterial stenosis. Am J Cardiol 1983; 51: 1462-3.

11. Rey C, Marache P, Francart C, Dupuis C. Percutaneous transluminal balloon valvuloplasty of congenital pulmonary valve stenosis, with a special report on infants and neonates. J Am Coll Cardiol 1988; 11: 815-20.

12. Narayanswami S, Saleem M, Jackson M, et al. Results of balloon pulmonary valvuloplasty as a palliative procedure in tetralogy of Fallot. J Am Coll Cardiol 1991; 18: 159-65.

13. Qureshi AS, Kirk CR, Lamb RK, Arnold R, Wilkinson JL. Balloon dilatation of the pulmonary valve in the first year of life in patients with tetralogy of Fallot: a preliminary study. Br Heart J 1988; 60: 232-5.

14. Ali Khan MA, Al-YousefS, Huhta JC, Bricker JT, Mullins CE, Sawyer W. Critical pulmonary valve stenosis in patients less than 1 year of age: treatment with percutaneous gradational balloon pulmonary valvuloplasty. Am Heart J 1989; 117: 1008-14.

15. Zeevi B, Keane JF, Fellows KE, Lock JE. Balloon dilation of critical pulmonary stenosis in the first week of life. J Am Coll Cardiol 1988; 11: 821-4.

16. Caspi J, Coles JG, Benson LN, et al. Management of neonatal critical pulmonic stenosis in the balloon valvotomy era. Ann Thorac Surg 1990; 49: 273-8.

17. Kveselis DA, Rocchini P, Snider R, Rosenthal A, Crowley DC, Dick M. Results of balloon valvuloplasty in the treatment of congenital valvar pulmonary stenosis in children. Am J Cardiol 1985; 56: 527-32.

18. Kan JS, White RI, Mitchell SE, Anderson JH, Gardner TJ. Percutaneous transluminal balloon valvuloplasty for pulmonary valve stenosis. Circulation 1984; 68: 554-60.

19. Stanger P, Cassidy SC, Girod DA, et al. Balloon pulmonary valvuloplasty: Results of the Valvuloplasty and Angioplasty of Congenital Anomalies Registry. Am J Cardiol 1990; 65: 775-83.

20. Lababidi Z, Wu J, Walls J. Percutaneous balloon Valvuloplasty: results in 23 patients. Am J Cardiol 1984; 53: 194-7.

21. Beheregaray $F^{\circ} \mathrm{LA}$, Zimmer LP, Velho FP,Zago AJ. Valvoplastia aórtica por balão no recém-nascido. Arq Bras Cardiol 1994; 63: 215-7.

22. Roberts WC. The congenitally bicuspid aortic valve. Am J Cardiol 1970; 26: 72-83.

23. Perloff JK. Estenose aórtica congênita: insuficiência aórtica congênita. In: Perloff JK. O Reconhecimento Clínico das Cardiopatias Congênitas. $3^{\mathrm{a}}$ Ed. São Paulo: Livraria Editora Santos, 1987: 92.

24. Ebaid M, Azeka E, Ikari NM, Atik E. Classificação e aproximação dignóstica. Rev Soc Cardiol Estado de São Paulo 1993; 3: 9-16.

25. Lababidi Z. Aortic balloon valvuloplasty. Am Heart J 1983; 106: 751.

26. HausdorfG, Schneider M, Schirmer K, Schulze-Neick I, Lange PE. Anterograde balloon valvuloplasty of aortic stenosis in children. Am J Cardiol 1993; 71: 460-2.

27. Shaddy RE, Boucek MM, Sturtevant JE, Ruttenberg HD, Orsmond GS. Gradient reduction, aortic valve regurgitation and prolapse after balloon aortic valvuloplasty in 32 consecutives patients with congenital aortic stenosis. J Am Coll Cardiol 1990; 16: 451-6.

28. Santana MVT, Silva MAP, Silva Jr JP. Indicação cirúrgica das cardiopatias congênitas acianogênicas. In: SOCESP Cardiologia. $2^{\circ}$ Vol. São Paulo: Atheneu, 1996: 843 .

29. Vobecky JS, Chartrand C, Angate H, Stanley P. Surgery for critical aortic stenosis in newborn is still a good therapy after 25 years. Can J Surg 1992; 35: 489-92.

30. Turley K, Bove EL, Amato JJ. Neonatal aortic stenosis. J Cardiovasc Surg 1991; 5:378-82.

31. Pena HPM, Teixeira SLC, Mandil A, Simão F ${ }^{\circ}$, Moreira MCV. Valvuloplastia aórtica por balão como ponte para o tratamento definitivo da estenose aórtica grave. Arq Bras Cardiol 1997; 69: 55-7.

32. NHLBI Balloon Valvuloplasty Registry Participants. Percutaneous balloon aortic valvuloplasty acute and 30-day follow-up results in 674 patients from the NHLBI balloon valvuloplasty registry. Circulation 1991; 84: 2383-97.

33. Rocchini AP, Beekman RH, Shachar GB, Benson L, Schwartz D, Kan JS. Balloon aortic valvuloplasty: results of the valvuloplasty and angioplasty of congenital anomalies registry. Am J Cardiol 1990; 65: 784-9. 
34. Souttar HS. The surgical tratment of mitral stenosis. Br Med J 1925; 2: 603-6.

35. Inoue K, Owaki T, Kitamura F, Miyamoto N. Clinical application of transvenous mitral commissurotomy by a new balloon catheter. J Thorac Cardiovasc Surg 1984; 87: 394-402.

36. Al Zaibag M, Ribeiro PA, Al Kasab S, Al Fagih MR. Percutaneous doubleballoon mitral valvotomy for rheumatic mitral valve stenosis. Lancet 1986; 1 : 757-61.

37. Alday LE, Juaneda E. Percutaneous balloon dilatation in congenital mitral stenosis. Br Heart J 1987; 57: 479-82.

38. Grifka RG, O'Laughlin MP, Nihill MR, Mullins CE. Double-transseptal, double-balloon valvuloplasty for congenital mitral stenosis. Circulation 1992; 85: $123-9$.

39. Ruckman RN, Van Praagh R. Anatomic types of congenital mitral stenosis: report of 49 autopsy cases with considerations of diagnostic and surgical implications. Am J Cardiol 1978; 42: 592-601.

40. Spevak P, Bass J, Bem-Shachar G, et al. Balloon angioplasty for congenital mitral stenosis. Am J Cardiol 1989; 66: 472-6.

41. Jarrar M, BetboutF, Gamra H, Maatouk F, Ayari M, Farhat MB. Successful percutaneous double balloon valvuloplasty for congenital mitral stenosis. Int J Cardiol 1996; 56: 193-6.

42. Fawzy ME, Mimish L, Awad M, Galal M, El-Deeb F, Khan B. Mitral balloon valvotomy in children with Inoue balloon technique; immediate and intermediateterm result. Am Heart J 1994; 127: 1559-62.

43. Ino T, Shimazaki S, Iwahara M, Miyazaki E, Yabuta K, Inoue K. Successful balloon mitral commissurotomy in a small child: use of small Inoue balloon catheter. Ann Thorac Surg 1993; 56: 168-70.

44. Essop MR, Govendrageloo K, Plessis JD, Van Dyk M, Sareli P. Balloon mitral valvotomy for rheumatic mitral stenosis in children aged $<12$ years. Am JCardiol 1993; 72: 850-1.

45. Carpentier A, Deloche A, Manania G, et al. Surgical management of acquired tricuspid valve disease. J Thorac Cardiovasc Surg 1974; 67: 53-65.

46. Kratz J. Avaliação e tratamento da doença valvar tricúspide. In: Clinicas Cardiológicas. Vol 2. Rio de Janeiro: Interlivros, 1991: 430.

47. Rubio-Alvarez V, Limon-Lason R. Comissurotomia tricuspidea por medio de un cateter modificado. Arch Inst Cardiol Méx 1955; 25: 57-69.

48. Al Zaibag M, Ribeiro P, Al Kasab S. Percutaneous balloon valvotomy in tricuspid stenosis. Br Heart J 1987; 57: 51-3.

49. Khalilullah M, Tyagi S, Yadav BS, Jain P, Choudhry A. Double-balloon valvuloplasty of tricuspid stenosis. Am Heart J 1987; 114: 1232.

50. Goldenberg IF, Pedersen W, Olson J, Madison JD, Mooney MR, Gobel FL. Percutaneous double balloon valvuloplasty for severe tricuspid stenosis. Am Heart J 1989; 118: 417-9.

51. Alberti A, Actis Dato A, Angelino A, Actis Dato A. Valvuloplastica tricuspidale percutanea. G Ital Cardiol 1994; 24: 1403-6.

52. Patel TM, Dani SI, Shah SC, Patel TK. Tricuspid balloon valvuloplasty: a more simplified approach using Inoue Balloon. Cathet Cardiovasc Diagn 1996; 37: 86-8.

53. Mullins PA, Hall JA, Shapiro LM. Balloon dilatation of tricuspid stenosis caused by carcinoid heart disease. Br Heart J 1990; 63: 249-50.

54. Becker AE, Becker MJ, Edwards JE. Anomalies associated with coarctation of the aorta. Circulation 1970; 41: 1067.

55. PerloffJK. Coarctação da aorta. In: PerloffJK. OReconhecimento Clínico das Cardiopatias Congênitas. $3^{\mathrm{a}}$ Ed. São Paulo: Livraria Editora Santos, 1987: 130.

56. Lock JE, Castaneda-Zuniga WR, Bass JL, Foker JE, Amplatz K, Anderson RW. Balloon dilatation of excised aortic coarctations. Radiology 1982; 143: 689-91.

57. Cooper RS, Ritter SB, Rothe WB, Chen CK, Griepp R, Golinko RJ. Angioplasty for coarctation of the aorta: long-term results. Circulation 1987; 75: 600-4.

58. Fontes VF, Esteves CA, Braga SLM, et al. It is valid to dilate native aortic coarctation with a balloon catheter. Int J Cardiol 1990; 27: 311-6.

59. Velasco ML, Reyes HR, Pérez TA, et al. Angioplastia transluminal percutanea de coartacion aortica en adolescentes y adultos. Resultados a mediano plazo. Arch Inst Cardiol Méx 1992; 62: 339-43.

60. De Lezo JS, Pan M, Romero M, et al. Balloon-expandable stent repair of severe coarctation of aorta. Am Heart J 1995; 129: 1002-8

61. Shaddy RE, Boucek MM, Sturtevant JE, et al. Comparison of angioplasty and surgery for unoperated coarctation of the aorta. Circulation 1993; 87: 793-9.

62. Fletcher SE, Nihill MR, Grifka RG, O'Laughlin MP, Mullins CE. Balloon angioplasty of native coarctation of the aorta: midterm follow-up and prognostic factors. J Am Coll Cardiol 1995; 25: 730-4.

63. Carvalho AC, Tebexreni AS, Silva C, Lima W. Aortoplastia com balão. Rev Soc Cardiol Estado de São Paulo 1995; 5: 486-93.

64. Isner JM, Donaldson RF, Fulton D, Bhan I, Payne DD, Cleveland RJ. Cystic medial necrosis in coarctation of the aorta: a potential factor contributing to adverse consequences observed after percutaneous balloon angioplasty of coarctation sites. Circulation 1987; 75: 689-95.

65. Smallhorn JF, Huhta JC, Adams PA, Anderson RH, Wilkinson JL, Macartney FJ.
Cross-sectional echocardiographic assessment of coarctation in the sick neonate and infant. Br Heart J 1983; 50: 349-61.

66. Mendelsohn AM, Lloyd TR, Crowley DC, Sandhu SK, Kocis KC, Beekman III RH. Late follow-up of balloon angioplasty in children with a native coarctation of the aorta. Am J Cardiol 1994; 74: 696-700.

67. Kulick DL, Kotlewski A, Hurvitz RJ, Jamison M, Rahimtoola SH. Aortic rupture following percutaneous catheter balloon coarctoplasty in an adult. Am Heart J 1990; 119: 190-3.

68. Park SC, Zuberbuhler JR, Neches WH, Lenox CC, Zoltun RA. A new atrial septostomy technique. Cathet Cardiovasc Diagn 1975; 1: 195-201.

69. Park SC, Neches WH, Zuberbuhler JR, et al. Clinical use of blade atrial septostomy. Circulation 1978; 58: 600-6.

70. Garcia DP, Macruz R, Constatino CF, Silva SS, Marcial MB. Técnica para septostomia interatrial através do cateterismo cardíaco. Arq Bras Cardiol 1973; 26: 515-7.

71. Atik E, Maeda W. Manuseio do recém-nascido cardiopata: o desafio persistente. Arq Bras Cardiol 1995; 64: 189-90.

72. Hausdorf G, Schulze-Neick I, Lange PE. Radiofrequency-assisted "reconstruction" of the right ventricular outflow tract in muscular pulmonary atresia with ventricular septal defect. Br Heart J 1993; 69: 343-6.

73. Qureshi AS, Rosenthal E, Tynan M, Anjos R, Baker EJ. Transcatheter laser-assisted balloon pulmonary valve dilation in pulmonic valve atresia. Am J Cardiol 1991; 67: 428-31.

74. Hausdorf G, Schneider M, Fink C, et al. Interventional right ventricular outflow tract creation in pulmonary atresia using radiofrequency. Cardiol Young 1993 3(suppl): 6 .

75. Piéchaud JF, Ladeia AM, Da Cruz E, et al. Perforation-dilatation des atrésies pulmonaires à septum interventriculaire intact chez le nouveau-né et le nourrisson. Arch Mal Coeur 1993; 86: 581-6.

76. Justo RN, Nykanen DG, Freedom RM, Benson LN. Outcomes of transcatheter perforation of the right ventricular outflow tract as primary management for pulmonary valve atresia in the newborn. Circulation 1995; 92(suppl I): I-380.

77. Fontes VF, Esteves CA, Braga SLN, et al. Atresia pulmonar com septo ventricular íntegro. Perfuração valvar por radiofreqüência. Arq Bras Cardiol 1995; 64: 231-3.

78. Cambier PA, Kirby WC, Wortham DC, Moore JW. Percutaneous closure of the small $(<2,5 \mathrm{~mm})$ patent ductus arteriosus using coil embolization. Am J Cardiol 1992; 69: 815-6.

79. Rao PS, Sideris EB. Transcatheter occlusion of patent ductus arteriosus: state of the art. J Invasive Cardiol 1996; 8: 278-88.

80. Gross RE, Hubbard JP. Surgical ligation of a patent ductus arteriosus. Report of the first successful case. JAMA 1939; 112: 729.

81. Krichenko A, Benson LN, Burrows P, Möes CAF, McLaughlin P, Freedom RM. Angiographic classification of the isolated, persistently patent ductus arteriosus and implications for percutaneous catheter occlusion. Am J Cardiol 1989; 63: $877-80$

82. Sato K, Fujino M, Kozuka T, et al. Transfemoral plug closure of patent ductus arteriosus. Experiences in 61 consecutive cases treated without thoracotomy. Circulation $1975 ; 51: 341$.

83. Rashkind WJ, Mullins CE, Hellenbrand WE, Tait MA. Nonsurgical closure of patent ductus arteriosus: clinical application of the Rashkind PDA occluder system. Circulation 1987; 75: 583-92.

84. Bridges ND, Perry SB, Parness I, Keane JF, Lock JE. Transcatheter closure of a large patent ductus arteriosus with the clamshell septal umbrella. J Am Coll Cardiol 1991; 18: 1297-302.

85. Pedra CAC, Pedra SRFF, Esteves CA, et al. Experiência global no fechamento percutâneo do canal arterial. Arq Bras Cardiol 1998; 71: 769-80.

86. Moore JW, George L, Kirkpatrick S, et al. Percutaneous closure of the small patent ductus arteriosus using occluding spring coils. J Am Coll Cardiol 1994; $23: 759-65$

87. Hijazi ZM, Geggel RL. Results of anterograde transcatheter closure of patent ductus arteriosus using single or multiple Gianturco coils. Am J Cardiol 1994; 74: 925-9.

88. Hosking MCK, Benson LN, Musewe N, Dick JD, Freedom RM. Transcatheter occlusion of the persistently patent ductus arteriosus. Forty-month follow-up and prevalence of residual shunting. Circulation 1991; 84: 2313: 7.

89. Report of the European Registry. Transcatheter Occlusion of Persistent Arterial Duct. Lancet 1992; 340: 1062-6.

90. Owada CY, Teitel DF, Moore PM. Evaluation of Gianturco coils for closure of large ( $\geq 3.5 \mathrm{~mm})$ patent ductus arteriosus. J Am Coll Cardiol 1997; 30: 1856-62.

91. Kajita LJ, Soares PJF, Medeiros CR, et al. Oclusão de canal arterial persistente por técnica percutânea: implante de coils de Gianturco. Rev Soc Cardiol Estado de São Paulo 1998; 8(4 supl B): 34.

92. Lloyd TR, Beekman III RH, Moore JW, etal. The PDA Coil Registry: report of the first 535 procedures. Circulation 1995; 92(suppl I): I-380.

93. Ladusans EJ, Murdoch I, Franciosi J. Severe haemolisis after percutaneous closure of a ductus arteriosus (arterial duct). Br Heart J 1989; 61: 548-50. 
94. Lloyd TR, Fedderly R, Mendelsohn AM, Sandhu SK, Beekman III RH. Transcatheter occlusion of patent ductus arteriosus with Gianturco coils. Circulation 1993; 88: 1412-20.

95. Kuhn MA, Latson LA. Transcatheter embolization coil closure of patent ductus arteriosus-modified delivery for enhanced control during coil positioning. Cathet Cardiovasc Diagn 1995; 36: 288-90.

96. Masura J, Walsh KP, Thanopoulous B, et al. Catheter closure of moderate-to largesized patent ductus arteriosus using the new Amplatzer duct occluder: immediate and short-term results. J Am Coll Cardiol 1998; 31: 878-82.

97. Zamorano MMB. Aspectos anatômicos das cardiopatias congênitas não cianogênicas. Rev Soc Cardiol Estado de São Paulo 1993; 3: 17-28.

98. Cockerman JT, Martin TC, Gutirrez FR, Hartman AF, Goldring D, Strauss AW. Spontaneous closure of secundum atrial septal defect in infants and young children. Am J Cardiol 1983; 52: 1268.

99. Ghisla RP, Hannon DW, Meyer RA, Kaplan S. Spontaneous closure of isolated secundum atrial septal defects in infants: an echocardiographic study. Am Heart J 1985; 109: 1327-33.

100. Mody MR. Serial hemodynamic observations in secundum atrial septal defect with special reference to spontaneous closure. Am J Cardiol 1973; 32: 978-81.

101. Fukazawa M, Fukushige J, Ueda K. Atrial septal defects in neonates with reference to spontaneous closure. Am Heart J 1988; 116: 123-7.

102. King TD, Mills NL. Nonoperative closure of atrial septal defects. Surgery 1974; 75: $383-8$.

103. Haddad J, Secches A, Finzi L, Carvalho S, Arévalo J. Oclusão percutânea transvenosa da comunicação interatrial. Rev Soc Cardiol Estado de São Paulo 1995; 5: 503-12.

104. Haddad J, Secches A, Finzi L, et al. Oclusão percutânea transvenosa de comunicação interatrial mediante utilização do buttoned device. Arq Bras Cardiol 1996; 67: 17-22.

105. Gildein HP, Dabritz S, Geibel A, et al. Transcatheter closure of atrial septal defects by the "buttoned" device: complications and need for surgical revision. Pediatr Cardiol 1997; 18: 328-31.

106. Formigari R, Santoro G, Rossetti L, Rinelli G, Guccione P, Ballerini L. Comparison of three atrial septal defect occlusion devices. Am J Cardiol 1998; 82: 690-2.

107. Walsh KP, Tofeig M, Kitchiner DJ, Peart I, Arnold R. Comparison of the Sideris and Amplatzer septal occlusion devices. Am J Cardiol 1999; 83: 933-6.

108. Thanopoulos BD, Laskari CV, Tsaousis GS, Zarayelyan A, Vekiou A, Papadopoulos GS. Closure of septal defects with the Amplatzer occlusion device: preliminary results. J Am Coll Cardiol 1998; 31: 1110-6.

109. Hausdorf G, Schneider M, Fink C, et al. Transcatheter closure of atrial septal defects within the oval fossa: medium-term results in children using the "ASDOS"technique. Cardiol Young 1998; 8: 462-71.

110. Masura J, Gavora P, Formanek A, Hijazi ZM. Transcatheter closure of secundum atrial septal defects using the new self-centering amplatzer septal occluder: initial human experience. Cathet Cardiovasc Diagn 1997; 42: 388-93.

111. Fontes VF, Pedra CAC, Pedra SRFF, et al. Experiência inicial no fechamento percutâneo da comunicação interatrial com a prótese de Amplatzer. Arq Bras Cardiol 1998; 70: 147-53.

112. Wilkinson JL, Goth TH. Early clinical experience with use of the "Amplatzer Septal Occluder"device for atrial septal defect. Cardiol Young 1998; 8: 295-302.

113. Soto B, Becker AE, Moulaert AJ, Lie JT, Anderson RH. Classification of ventricular septal defects. Br Heart J 1980; 43: 332-43.

114. PerloffJK. Defeito septal ventricular. In: PerloffJK. O Reconhecimento Clínico das Cardiopatias Congênitas. $3^{a}$ Ed. São Paulo: Livraria Editora Santos, 1987: 371.

115. PerloffJK. Defeito septal ventricular. In: PerloffJK. O Reconhecimento Clínico das Cardiopatias Congênitas. 3ª Ed. São Paulo: Livraria Editora Santos, 1987: 375.

116. Gersony WM, Hayes CJ. Bacterial endocarditis in patients with pulmonary stenosis, aortic stenosis, or ventricular septal defect. Circulation 1977; 56: I-84-I-87.

117. Lock JE, Block PC, McKay RG, Baim DS, Keane JF. Transcatheter closure of ventricular septal defects. Circulation 1988; 78: 361-8.
118. Bridges ND, Perry SB, Keane JF, et al. Preoperative transcatheter closure of congenital muscular ventricular septal defects. N Engl J Med 1991; 324: 1312-7.

119. Rigby ML, Redington NA. Primary transcatheter umbrella closure of perimembranous ventricular septal defect. Br Heart J 1994; 72: 368-71.

120. Sideris EB, Walsh KP, Haddad JL, Chen CR, Ren SG, Kulkarni H. Occlusion of congenital ventricular septal defects by the buttoned device. Heart 1997; 77: 276-9.

121. Thanopoulos BD, Tsaousis GS, Konstadopoulou GN, Zarayelyan AG. Transcatheter closure of muscular ventricular septal defects with the Amplatzer ventricular septal defect occluder: initial clinical applications in children. J Am Coll Cardiol 1999; 33: 1395-9.

122. Dotter CT. Transluminalally-placed coil spring endarterial tube grafts: long term patency in canine popliteal artery. Invest Radiol 1969; 4: 329-32.

123. Sigwart U, Puel J, Mirkovitch V, Joffre F, Kappenberger L. Intravascular stents to prevent occlusion and restenosis after transluminal angioplasty. N Engl J Med 1987; 316: 701-6.

124. SchatzRA, Palmaz JC, Tio FC, Garcia F, Garcia O, Reuter SR. Balloon expandable intracoronary stents in the adult dog. Circulation 1987; 76: 450-7.

125. Morrow WR, Smith VC, Ehler WJ, VanDellen AF, Mullins CE. Balloon angioplasty with stent implantation in experimental coarctation of the aorta. Circulation 1994; 89: 2677-83.

126. Ino T, Shimazaki S, Yabuta K, Okada R. Experimental studies on the implantation of intravascular stents in puppies - potential use for aortic coarctation and pulmonary arterial stenosis. Cardiol in the Young 1994; 4: 366-72.

127. Diethrich EB, Heuser RR, Cardenas JR, Eckert J, Tarlian H. Endovascular techniques in adult aortic coarctation: the use of stents for native and recurrent coarctation repair. J Endovasc Surg 1995; 2: 183-8.

128. BulbulZR, Bruckheimer E, Love JC, Fahey JT, Hellenbrand WE. Implantation of balloon-expandable stents for coarctation of the aorta: implantation data and short-term results. Cathet Cardiovasc Diagn 1996; 39: 36-42.

129. Pedulla DM, Grifka RG, Mullins CE, Allen D. Endovascular stent implantation for severe recoarctation of the aorta: case report with angiographic and 18-month clinical followw-up. Cathet Cardiovasc Diagn 1997; 40: 311-4.

130. Ledesma M, Diazy Diaz E, Alva EC, et al. Stents en coarctación aórtica. Arch Inst Cardiol Méx 1997; 67: 399-404.

131. Ebeid MR, Prieto LR, Latson LA. Use of balloon-expandable stents for coarctation of the aorta: initial results and intermediate-term follow-up. J Am Coll Cardiol 1997; 30: 1847-52.

132. De Lezo JS, Pan M, Romero M, et al. Immediate and follow-up findings after stent treatment for severe coarctation of aorta. Am J Cardiol 1999; 83: 400-6.

133. Radtke WA. Interventional pediatric cardiology: state of the art and future perspective. Eur J Pediatr 1994; 153: 542-7.

134. Powell AJ, Lock JE, Keane JF, Perry SB. Percutaneous of RV-PA conduit life span by percutaneous stent implantation. Intermediate-term results. Circulation 1995; 92: 3282-8

135. Alcibar J, Ramon RJ, Cabrera A, etal. Experience with Palmaz stent in pulmonary branch stenosis. Rev Esp Cardiol 1997; 50: 179-86.

136. Hijazi ZM. Stenting for postoperative congenital heart disease in infants. Cathet Cardiovasc Diagn 1997; 42: 195.

137. Coulson JD, Bullaboy CA. Concentric placement of stents to relieve an obstructed anomalous pulmonary venous connection. Cathet Cardiovasc Diagn 1997; 42: 201-4

138. Schneider M, Zartner P, Sidiropoulos A, Konertz W, HausdorfG. Stent implantation of the arterial duct in newborns with duct-dapendent circulation. Eur Heart J 1998; 19: 1401-9.

139. Peuster M, Fink C, Bertram H, Paul T, Hausdorf G. Trancatheter recanalization and subsequent stent implantation for the treatment of early postoperative thrombosis of modified Blalock-Taussig shunts two children. Cathet Cardiovasc Diagn 1998; 45: 405-8. 